\title{
Uso de objetos de aprendizagem digital para flexibilizar o conhecimento e potencializar a autonomia do aprendizado no ensino da educação ambiental
}

\author{
Use of learning objects digital for flexibilize the knowledge and potentialize \\ the autonomy of learning in teaching of environmental education \\ Deise Quiele Rauber Fiuza', Liziany Muller², Arruda Pereira Arruda ${ }^{3}$, \\ Andreia Oliveira Machado ${ }^{4}$ \\ I, 2, 3,4 Universidade Federal de Santa Maria, Santa Maria, RS, Brasil
}

\section{Resumo}

Apesar da relevância da Educação Ambiental (EA) na educação formal, ela ainda se encontra fragilizada em sua prática pedagógica, e muito contingente no uso das tecnologias. Neste sentido, este estudo propõe avaliar a contribuição e as potencialidades do uso de objetos de aprendizagem digital (OAD) no ensino da Educação Ambiental, envolvendo alunos do Ensino Fundamental II do Colégio Franciscano Sagrado Coração de Jesus, no município de Arroio do Tigre. O uso das tecnologias pode trazer inúmeras contribuições para a educação, como a autonomia do aluno no processo de aprendizado bem como a sua inclusão digital. Os ODA propõem o uso de atividades de conscientização por meio do uso da tecnologia com atividades práticas virtuais, abordando temas relacionados com a questão ambiental, sustentabilidade, moda, consumismo e lixo. Partindo de um pressuposto local, porém não deixando de considerar o ambiente em seus múltiplos aspectos, com uma visão ampla de alcance regional e global, foi aplicado o cálculo da pegada ecológica e construídos vídeos de educação ambiental para serem espalhados virtualmente nas redes sociais e no Youtube.

Palavras-chave: Educação; Educação Ambiental; Objetos de Aprendizagem Digital; Pegada Ecológica; Tecnologia Educacional.

\begin{abstract}
Despite the importance of environmental education (EE) in formal education, it is still weak in teaching practice and much contingent on the use of technology. Thus, this study aims to evaluate the contribution and on the potential use of digital learning objects in teaching environmental education, involving elementary school students of the Colégio Franciscano Sagrado Coração de Jesus, in Arroio do Tigre city. The technology use can result in several contributions to education, such as: student autonomy in the learning process and their inclusion in the digital world. The learning objects propose the use of digital awareness activities through the use of technology with virtual practical activities related to environmental issues, sustainability, fashion, consumerism and waste. Starting from a premise location, but not leaving consider the environment in its multiple aspects, with a broad range of regional and global, was applied to calculate the carbon footprint and environmental education videos built to be virtually spread social networks and Youtube.
\end{abstract}

Keywords: Education; Environmental Education; Digital Learning Objects; Ecological Footprint; Technology educational. 


\section{INTRODUÇÃO}

Os Parâmetros Curriculares Nacionais (PCNs, 1997) apresentam a questão ambiental como um dos temas transversais do currículo do Ensino Fundamental, e recomenda que o processo educativo deve romper com o adestramento e a simples transmissão de conhecimentos, destacando que cada professor, dentro da especificidade de sua área, deve adequar o tratamento dos conteúdos para contemplar o tema Meio Ambiente, assim como os demais temas transversais.

A Educação Ambiental pode ser definida como um processo que consiste em propiciar às pessoas uma compreensão crítica e global do meio ambiente, procurando elucidar valores e atitudes na adoção de posturas éticas e participativas nas questões relacionadas à conservação e adequada utilização dos recursos naturais (NEVES, 2003).

Nesse contexto, as tecnologias da informação e da comunicação (TIC) apresentam diversas possibilidades de se trabalhar essa temática. Conforme aponta Hamze (2010), quando o professor desenvolve competências e habilidades para trabalhar com recursos tecnológicos, contextualizando suas atividades didáticas, esses procedimentos serão usados como mais uma ferramenta pedagógica enriquecedora do texto e do contexto que estão sendo trabalhados. Assim sendo, a tecnologia surge para incrementar não só a carreira do docente, como também a de muitos outros profissionais, podendo contribuir para o processo de aprendizagem, porém, é preciso saber explorá-la para obter bons resultados (LAZARINI, 2010), pois, conforme afirmam Sampaio e Leite (1999), não basta a simples utilização de tecnologia, é necessário inovar em termos de prática pedagógica.

$\mathrm{O}$ avanço da tecnologia permitiu que o acesso à informação se tornasse muito mais rápido e fácil (ALMEIDA \& PRADO, 2009). As atuais tecnologias de informação e comunicação (TIC) apresentam novas possibilidades para o indivíduo vivenciar processos criativos, estabelecendo aproximações e associações inesperadas, juntando significados anteriormente desconexos e ampliando a capacidade de interlocução por meio das diferentes linguagens que tais recursos propiciam (MARTINSI, 2008).

Nesse sentido, Kenski (2010) afirma que

A velocidade das alterações no universo informacional exige atualização permanente, pois as tecnologias estão em permanente mudança, à aprendizagem por toda a vida torna-se consequência natural do momento social e tecnológico em que vivemos, a sensação é de que quanto mais aprendemos mais se tem a estudar para se atualizar (KENSKI, 2010).

Nessa concepção, um OAD pode ser um potencializador para a configuração de significações que venham a conformar a aprendizagem dos alunos, além de desempenhar uma função social, pois proporcionam a inclusão digital de muitos alunos e muito conhecimento. (SANTOS, 2008).

Nesse contexto, Nitzke (2002) afirma que

É preciso criar situações para que esse aluno estabeleça relações. Para que estabeleça relações entre relações, que faça construções renovadas e reinvente as noções que se pretende que ele aprenda. "Só assim se alcança a compreensão de um conhecimento (NITZKE,2002).

A ação crítica dos sujeitos do processo educacional e a tomada de decisões nessa nova realidade é apontada por Freire (2005). Para o autor, a educação deve ajudar o homem brasileiro a inserir-se criticamente no processo histórico e libertar-se, pela conscientização, da síndrome do ter e da escravidão do consumismo.

Cada vez que se observa notícias sobre a crise ambiental em jornais, revistas, na televisão ou no rádio, o ser humano, em geral, não percebe a relação entre o seu cotidiano e a situação do planeta. Muitas vezes, remete à responsabilidade nos outros sem olhar para os seus hábitos nas suas casas, trabalhos e comunidades.

Na tentativa de reverter à situação do planeta, o WWF-Brasil, uma organização não-governamental dedicada a Educação Ambiental, criou um OAD "Cálculo da Pegada Ecológica" que permite perceber que a trajetória de cada ser humano no planeta deixa "marcas", "pegadas", de acordo com a forma como se caminha.

A Pegada Ecológica ou Ecological Footprint (EF) é uma ferramenta de avaliação, proposta por Wackernagel e Rees (1996), que representa o espaço ecológico necessário para sustentar um determinado sistema ou unidade. Trata-se de um instrumento que contabiliza os fluxos de matéria e energia que entram e saem de um sistema econômico, convertendo-os em área correspondente de Planeta Terra, diretamente relacionados ao desenvolvimento sustentável, ao uso racional e equitativo dos recursos naturais (VAN BELLEN, 2006). 
Com base nos pressupostos apresentados, este trabalho visa avaliar à utilização de Objetos de Aprendizagem Digital (OAD), por alunos de $8^{\circ}$ e $9^{\circ}$ ano do Ensino Fundamental, com o objetivo de potencializar as aulas de Educação Ambiental através de tecnologias educacionais inovadoras, como promotoras da autonomia do aprendizado no desenvolvimento psíquico-intelectual dos estudantes, na busca de uma aprendizagem significativa sobre a conscientização e a preservação ambiental.

\section{MATERIAIS E MÉTODOS}

O projeto foi desenvolvido no Colégio Franciscano Sagrado Coração de Jesus, da rede particular de ensino, localizado na Rua Dom Guilherme Müller, 1075, centro, na cidade de Arroio do Tigre, Rio Grande do Sul, Brasil. O município possui cerca de 13 mil habitantes e a principal atividade econômica é a agricultura com destaque na produção de fumo, milho e feijão, sendo um dos maiores produtores no Rio Grande do Sul. O Colégio atende a comunidade local e regional, fundado em 17 de fevereiro de 1928 pelas Irmãs Franciscanas de Bonlanden que até hoje mantém o comando da Instituição, que faz parte da Rede Católica de Ensino. Atualmente possui 217 alunos e 22 professores que atuam desde a Educação Infantil a partir dos 2 anos, Pré Escolar (A e B), Ensino Fundamental I $\left(1^{\circ}\right.$ ao $5^{\circ}$ ano), Ensino Fundamental II ( $6^{\circ}$ ao $9^{\circ}$ ano), Ensino Médio com Técnico Normal e Curso Técnico em Enfermagem.

A Instituição tem como objetivo acreditar na pessoa humana, promovendo a educação humana e cristã que se fundamenta no amor e na liberdade que possibilita ao aluno tornar-se cidadão comprometido com a vida e com a história, abrindo-se à transcendência com explicação definitiva de seu existir. A instituição proporciona também aos estudantes situações que contribuam para o desenvolvimento social, cognitivo, afetivo, psicomotor, atenção e o raciocínio lógico, dentro de uma pedagogia lúdica e de livre expressão, levando-os à estruturação das noções básicas na formação da sua personalidade e de caráter capazes de auxiliá-los na leitura e na escrita do mundo.

O Colégio dispõe de um laboratório de informática com 20 computadores com acesso à internet em toda Instituição, possui também, uma Lousa Digital que proporciona maior interação entre os alunos e professores e 2 salas com Data Show. Além disso, dispõe de 8 computadores para pesquisa na biblioteca, 2 salas de vídeo, microscópico eletrônico no laboratório de Ciências e a sala RCE SAT que representa a rede de satélite da Rede Católica de Educação. Todas as suas escolas integradas receberam as antenas satélites e participam ativamente do extenso planejamento, que possibilita a interação direta entre apresentador, palestrante e plateia, via telefone, chat e e-mail, em tempo real.

O Projeto teve como sujeitos de pesquisa alunos do Ensino Fundamental II ( $8^{\circ}$ e $9^{\circ}$ ano). A utilização dos Objetos de Aprendizagem Digital (OAD) se deu em algumas etapas, primeiramente, os estudantes assistiram na Lousa digital do Colégio os seguintes documentários: "A História das Coisas", 20 minutos, disponível em: http://www.youtube.com/watch=v=ZpkxCpxKilIHYPERLINK "http:// www.youtube.com/watch?v=ZpkxCpxKilI\&feature=fvst" \&HYPERLINK "http://www.youtube.

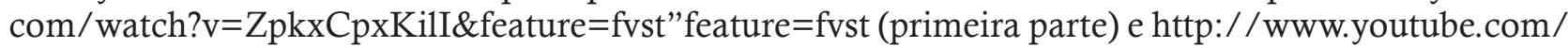
watch?v=ZgyNw5pIXE8HYPERLINK "http://www.youtube.com/watch?v=ZgyNw5pIXE8\&featur $\mathrm{e}=$ relmfu" \&HYPERLINK "http://www.youtube.com/watch?v=ZgyNw5pIXE8\&feature=relmfu"fe ature=relmfu, (segunda parte). e o "Planeta Faminto" (capítulo 2), 7 minutos, disponível em: http:// youtu.be/6zWIAtWXNY0. Os vídeos retratam o atual avanço tecnológico que se encontra nossa sociedade e a necessidade de se pensar e desenvolver mais a Educação Ambiental em nosso cotidiano.

$\mathrm{Na}$ próxima etapa, aplicou-se, no laboratório de informática do Colégio, o OAD "Cálculo da Pegada Ecológica" com as duas turmas de estudantes. Este OAD é composto por um questionário que se encontra no Anexo A (Figuras 05 ao 10) que calcula a pegada ecológica de cada indivíduo, fazendo uma estimativa da quantidade da área do planeta necessária a cada ser humano para prover a demanda por produtos e serviços consumidos pela população. Para realização do cálculo, os alunos acessaram o site individualmente: http://www.footprintnetwork.org/en/index.php/ GFN/page/calculators/ http://ecobairro .org.br/site/pegada.pdf.

Como auxílio dos OAD utilizados, realizou-se discussões em sala de aula e também pesquisas na internet sobre temas relacionados à Educação Ambiental com destaque para o consumismo. A partir disso, no laboratório de informática do Colégio, os alunos separados por turmas construíram vídeos 
em duplas e trios no programa Windons Movie Maker, que para muitos alunos foi a primeira vez que realizaram um trabalho nesse programa, e, posteriormente, postaram nas redes sociais e no Youtube (www.facebook.com.br e www.youtube.com.br).

As turmas do $8^{\circ}$ e $9^{\circ}$ ano construíram vídeos relacionados com a temática do lixo, consumismo, moda e a degradação do meio ambiente, os vídeos construídos estão nos seguintes endereços: http://www. youtube.com/watch?v=OCJo_e LPRRoHYPERLINK "http://www.youtube.com/watch?v=OCJo_e\%20 LPRRo\&feature $=\% 20$ share\&list\%20=ULOCJo_eLPRRo" \&HYPERLINK "http://www.youtube.com/ watch?v=OCJo_e\%20LPRRo\&feature $=\% 20$ share\&list $\% 20=$ ULOCJo_eLPRRo"feature $=$ shareHYPERLINK "http://www.youtube.com/watch?v=OCJo_e\%20LPRRo\&feature=\%20share\&list $\% 20=$ ULOCJo_eLPRRo"\&HYPERLINK "http://www.youtube.com/watch?v=OCJo_e\%20LPRRo\&feature=\%20 share\&list $\% 20=$ ULOCJo_eLPRRo"list =ULOCJo_eLPRRo (Figura 07), http://www.youtube.com/ watch?v=juFGeUROy8HYPERLINK "http://www.youtube.com/watch?v=juFGeUROy8\&feature= BFa\&list=ULOCJo_eLPRRo"\&HYPERLINK "http://www.youtube.com/watch?v=juFGeUROy8\& feature $=$ BFa\&list=ULOCJo_eLPRRo"feature=BFaHYPERLINK "http://www.youtube.com/watch? $\mathrm{v}=$ juFGeUROy8\&feature=BFa\&list=ULOCJo_eLPRRo" \&HYPERLINK "http://www.youtube.com/ watch?v=juFGeUROy8\&feature=BFa\&list=ULOCJo_eLPRRo"list=ULOCJo_eLPRRo (Figura 08), e os demais vídeos se encontram no Anexo B (Figuras 11 ao 19).
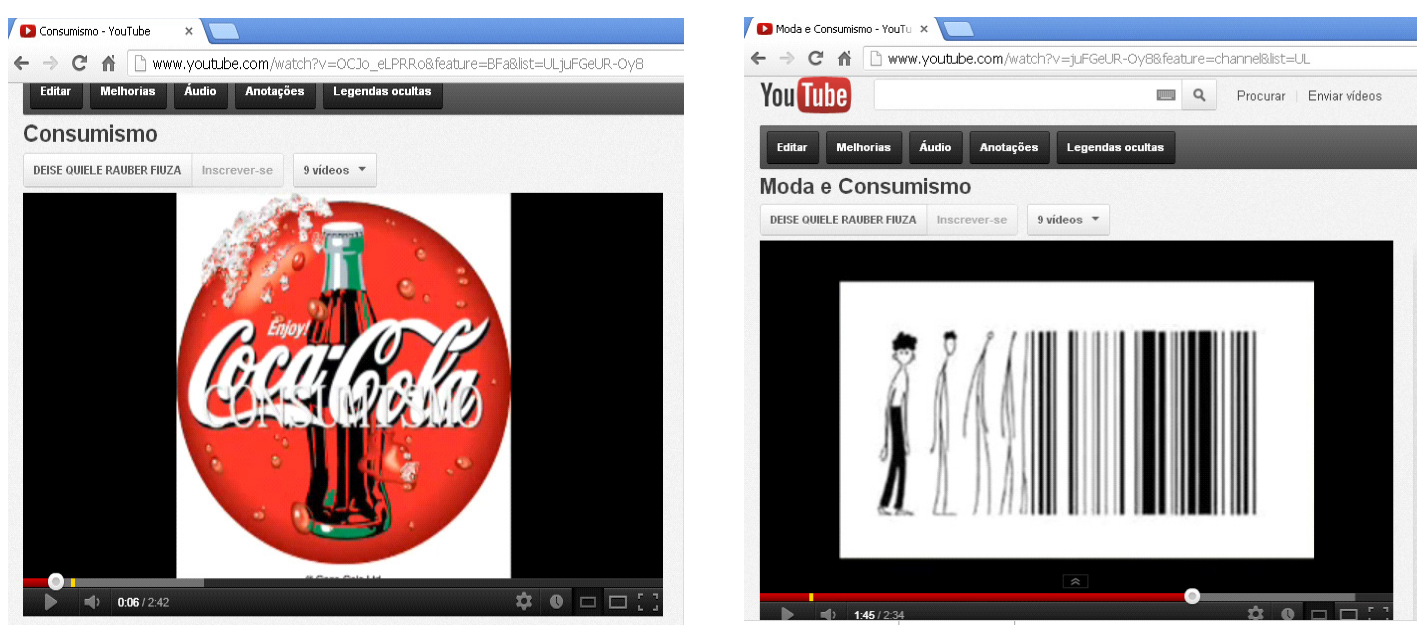

Figura 01 e 02: Vídeos construídos pelos alunos do $8^{\circ}$ e $9^{\circ}$ ano.

Os vídeos também foram divulgados a comunidade escolar numa sessão de apresentação na Lousa Digital para todos os alunos do Ensino Fundamental e Ensino Médio Normal, que juntamente com os colegas e professores da Instituição debateram sobre Educação Ambiental e a necessidade de mudanças de hábitos em nosso cotidiano. A proposta de construção dos vídeos teve como objetivo demonstrar a atual situação em que se encontra nosso planeta, visando uma maior interação e conscientização com outros sujeitos em rede sobre a EA.

\section{ANÁLISE DE DADOS E DISCUSSÃO}

Após aplicação do projeto a partir dos $\mathrm{OAD}$, principalmente através do cálculo da pegada ecológica, os alunos, de uma forma geral, tomaram consciência de que ações individuais podem prejudicar o nosso planeta. Com o cálculo da pegada ecológica foi possível perceber que são necessárias mudanças de hábitos em nosso cotidiano, uma vez que, nas duas turmas, mais de $50 \%$ dos alunos necessitaram de mais de um planeta para sua sobrevivência.

A pegada ecológica de um país é a área total requerida para a produção de todas as demandas de consumo de sua população, incluindo alimentação, vestuário, educação, saúde, cultura, trabalho, moradia, transporte, comunicação, entretenimento, etc., as quais implicam exploração da natureza no que diz respeito à matéria prima, a energia, a água, a terras agricultadas, a áreas urbanizadas e, ainda, 
a bolsões de absorção dos resíduos gerados por todas as etapas implicadas neste processo antrópico (DIAS, 2004).

Portanto, em decorrência do ato de consumir produtos e serviços diariamente, a população mundial consome componentes ecológicos do planeta como um todo, de modo que a pegada ecológica da humanidade é a soma de todas essas áreas implicadas, onde quer que elas estejam no planeta. Com base no cálculo realizado observa-se os resultados obtidos nas seguintes tabelas e gráficos: Tabela 1. Lista da turma do $8^{\circ}$ ano com o número de planetas que necessitam.

\begin{tabular}{|c|c|}
\hline $\begin{array}{l}\text { Número de alunos } \\
\text { Total: } 14 \text { alunos }\end{array}$ & Número de Planetas \\
\hline 10 & 1,1 \\
\hline 04 & 0,9 \\
\hline
\end{tabular}

Tabela 2. Lista da turma do $9^{\circ}$ ano com o número de planetas que necessitam.

\begin{tabular}{|c|c|}
\hline $\begin{array}{l}\text { Número de alunos } \\
\text { Total: } 11 \text { alunos }\end{array}$ & Número de Planetas \\
\hline 07 & 1,1 \\
\hline 04 & 0,8 \\
\hline
\end{tabular}

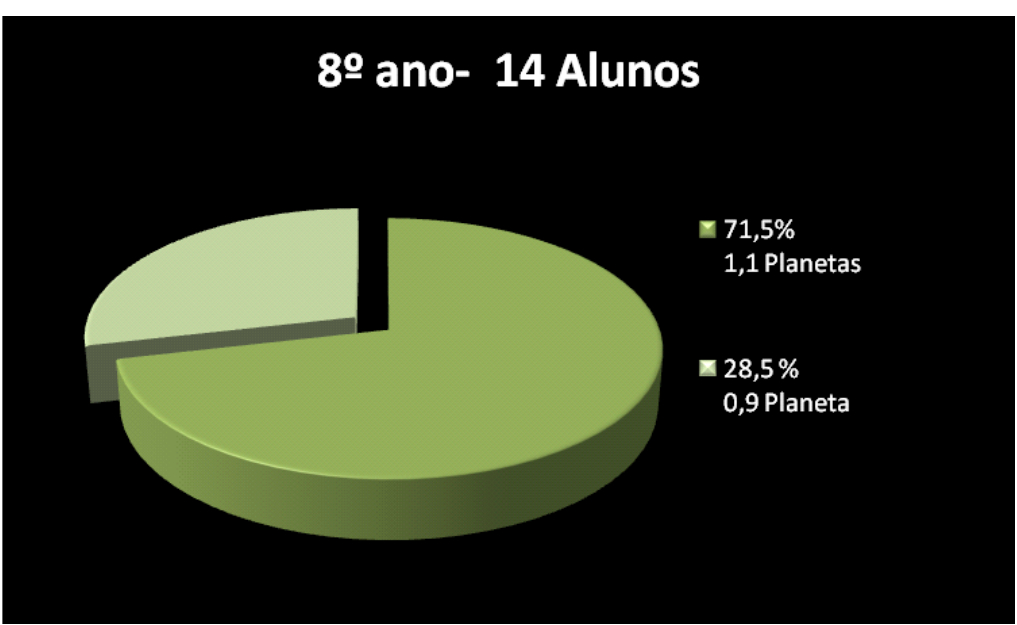

Figura 03: Gráfico da turma do $8^{\circ}$ ano com o percentual dos alunos e a quantidade de planetas necessários para sua sobrevivência.

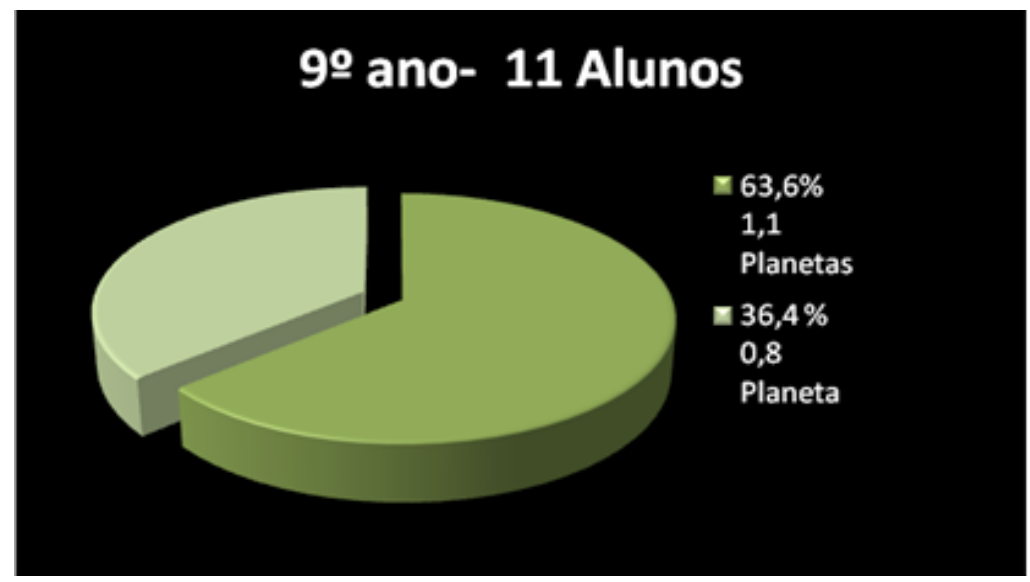

Figura 04: Gráfico da turma do $9^{\circ}$ ano com o percentual dos alunos e a quantidade de planetas necessários para sua sobrevivência. 
Nos gráficos apresentados (tabelas e figuras) pode-se observar um percentual bem elevado do número de planetas para a sobrevivência de cada aluno. Na tabela e no gráfico da figura 03 , da turma do $8^{\circ}$ ano, demonstra-se que, dos 14 alunos, $71,50 \%$ necessitam de 1,5 Planetas e 28,50\% necessitam de 0,9 Planetas Terra para sua sobrevivência. Na tabela e no gráfico da figura 04 , da turma do $9^{\circ}$ ano, demonstra-se que, do total de 11 alunos, $63,6 \%$ necessitam de 1,1 Planetas e $36,4 \%$ necessitam de 0,8 Planeta Terra para sua sobrevivência.

Temos disponíveis, na Terra, 1,8 hectares por pessoa, segundo o site Global Footprint Network : http://www.footprintnetwork.org/en/index.php/GFN/, além de que, obviamente, temos apenas um planeta disponível para vivermos, se todos no mundo consumissem como nós brasileiros, seriam necessários 1,16 planetas Terras. Nesse contexto, percebemos que o consumo brasileiro já se tornou insustentável, e o planeta já não é capaz de suprir nossas necessidades. Segundo o mesmo site sobre o consumo mundial atual, toda a população necessitaria de 1,5 planetas Terras disponíveis para sobreviver. A não ser que encontrem outro planeta, em breve o nosso se esgotará.

Como mostra os resultados obtidos no OAD "Cálculo da Pegada Ecológica", quando mais se acelera nossa exploração do meio ambiente, maior se torna a marca que deixamos na Terra. Ele faz uma estimativa e mostra até que ponto a forma de viver do ser humano esta de acordo com a capacidade do planeta de oferecer, renovar seus recursos naturais e absorver os resíduos que geramos por muitos e muitos anos.

De acordo com os Parâmetros Curriculares Nacionais para o Ensino Fundamental (PCNs, 1997) a Educação Ambiental (EA) deve ser apreendida em suas relações com a tecnologia e com as demais questões sociais e ambientais. Nesse contexto, as TIC surgem entre os temas atuais que precisam fazer parte do processo de ensino-aprendizagem e apresentam diferentes possibilidades de se trabalhar essa temática. A construção dos vídeos sobre Educação Ambiental (Anexo B) proporcionou uma maior interação entre os educandos e o meio tecnológico, com a aquisição de valores e conhecimentos, atitudes e conscientização responsável.

Percebe-se que os métodos de ensino convencionais já não agradam muito os estudantes de hoje, para conseguir despertar a atenção dos estudantes é preciso estar atento ao seu cotidiano e, mais, integrado com as mudanças tecnológicas. A tecnologia enriquece a aula, quando utilizada como uma alternativa no ensino dos conteúdos, pois facilita a aprendizagem, motiva o envolvimento e a participação do aluno e reduz o tempo deste processo de aprendizado.

O uso dos meios tecnológicos precisa ser utilizado como uma ferramenta de mediação no processo de ensino-aprendizagem. Na opinião de Enricone (2006) a boa tecnologia é aquela que permite o crescimento pessoal e a autoformação dos aprendentes.

De acordo com Gallota e Nunes (2004), inúmeras são as contribuições, que um OAD pode proporcionar à aprendizagem, pois além de contextualizar um determinado assunto, são meios eficientes no sentido de proporcionar a visualização de conceitos complexos, além de induzir o pensamento e a interpretação. Para esses autores, outra vantagem da utilização de OAD é a interatividade que eles propiciam, estes incentivam a participação do aluno permitindo um papel ativo, diferente de quando o aluno apenas é um ouvinte e assume uma posição passiva das informações (GALLOTTA; NUNES, 2004).

Torna-se evidente que sem um processo educativo consistente e participativo, que consiga abranger toda a sociedade, é inviável a busca pela sociedade sustentável. Fez-se necessário captar as representações de sociedade, educação, ambiente, natureza, indivíduo-sociedade, escola e, finalmente cidadania, que os envolvidos tinham concreta e simbolicamente construídas ao longo e no cotidiano de suas vidas. O desafio posto configurou-se em mobilizar tais representações para o debate e ampliação de outras mais próximas da realidade desejada e objetivada no projeto.

\section{CONSIDERAÇÕES FINAIS}

No ensino da Educação Ambiental, foco da pesquisa deste artigo, inovações tecnológicas desta natureza tornam-se urgentes, pois exige-se cada vez mais, atitudes conscientes com potencial criativo, sujeitos que saibam tomar decisões e resolver problemas. Neste contexto, as TIC podem contribuir significativamente, tornando o processo de ensino-aprendizagem mais dinâmico e participativo. As 
ferramentas disponíveis atualmente, como destaque os objetos de aprendizagem virtual que apresentou os vídeos construídos pelos alunos sobre Educação Ambiental se tornaram recursos digitais virtuais de ensino-aprendizagem para muitos sujeitos.

A introdução da Educação Ambiental nos diversos graus de ensino se faz necessária e é de fundamental importância, pois ela sensibiliza e forma futuros cidadãos conscientes. Com desenvolvimento das ações deste projeto, foi possível constatar a real necessidade de mudar os atos consumistas, para posteriormente não acabar destruindo nosso planeta. Com a realização do cálculo da pegada ecológica e da construção dos vídeos que foram postados nas redes sociais e no Youtube sobre diferentes temas ligados à moda, ao consumismo, ao lixo e à Educação Ambiental, houve uma interação sobre o assunto com pessoas de diferentes lugares e constatou-se que é imprescindível e necessário o uso dos meios tecnológicos para desenvolvimento de atividades de conscientização e Educação Ambiental. Os objetos de aprendizagem digitais potencializaram o ensino da Educação Ambiental e buscaram cada vez mais despertar a consciência ambiental responsável nos educandos.

\section{REFERÊNCIAS}

ALMEIDA, M.E.B; PRADO, M.E.B.B. Integração tecnológica, linguagem e representação. 2009. Disponível em: http://midiasnaeducacao-joanirse.blogspot.com/2009/02/integracao-tecnologica-linguagem-e. htmHYPERLINK "http://midiasnaeducacao-joanirse.blogspot.com/2009/02/integracao-tecnologica-linguagem-e.html"1. Acesso em 14 de out. 2012

DIAS, Genebaldo Freire. Ecopercepção- Um resumo didático dos desafios socioambientais. Editora Gaia, 2004.

ENRICONE, D. A dimensão pedagógica da prática docente futura. In: ENRICONE, D. (orgs). A docência na educação superior- sete olhares. Porto Alegre: Evangraf, 2006.

FREIRE, Paulo. Pedagogia do oprimido. Rio de Janeiro. Paz e Terra, 2005.

GALLOTTA, Alexandre; NUNES, César Augusto. Objetos de aprendizagem a serviço do professor, 2004. Disponível em: <www.microsoft.com/brasil/ educacao/parceiro/objeto_texto.mspx>. Acesso em: 12 out. 2012.

Global Footprint Network . Site. Disponível em: http://www.footprintnetwork. org/en/index.php/GFN/. Acesso em 23 de out. de 2012.

HAMZE, A. Linguagem Audiovisual e a Educação. 2010. Disponível em: http://www.educador.brasilescola. com/gestao-educacional/linguagem.htm. Acesso em 13 de out. de 2012.

HISTÓRIA DAS COISAS. Versão brasileira do documentário The Story of Stuff, de Annie Leonard, 20min. Disponível em: http://www.youtube.com/watchHYPERLINK "http://www.youtube.com/watch?v=ZpkxC pxKilI\&feature $=$ fvst" $=$ HYPERLINK "http://www.youtube.com/watch? $v=Z$ pkxCpxKilI\&feature $=$ fvst" $v=Z$ pkxCpxKilIHYPERLINK "http://www.youtube.com/watch?v=ZpkxCpxKill\&feature=fvst" \&HYPERLINK "http://www.youtube.com/watch?v=ZpkxCpxKilI\&feature=fvst"feature=fvst (primeira parte) e http://www. youtube.com/watch?v=ZgyNw5pIXE8HYPERLINK "http://www.youtube.com/watch?v=ZgyNw5pIXE8\&f eature=relmfu" \&HYPERLINK "http://www.youtube.com/watch?v=ZgyNw5pIXE8\&feature=relmfu"featur e=relmfu, (segunda parte). Acesso em 10 de out. de 2012.

KENSKI, V.M. Educação e tecnologias o novo ritmo da informação. Campinas. Papirus, 6 ed. 2010 v.1. 141 p.

LAZARINI, S. Utilizando a tecnologia a seu favor. Disponível em: http://www.universia.com.br/materia/ imprimir.jsp?id=11941. Acesso em: 09 out. de 2012. 
MARTINSI. M.C. Situando o uso da mídia em contextos educacionais. 2008. Disponível em: http://midiasnaeducacao-joanirse.blogspot.com/2008/12/ situando-o-uso-da-mdia-em-contextos.html. Acesso em: 20 de out. de 2010.

NEVES, S. Proposta de educação sócio-ambiental: Educação para o futuro. Uruaçu. 2003. Disponível em: $<$ http//www.semaar.pi.gov.br/>.Acesso em:08 out. 2012.

NITZKE, J. A. CARNEIRO, M. L. F. FRANCO, S. R. K. Ambientes de Aprendizagem Cooperativa Apoiada pelo Computador e sua Epistemologia. In: Informática na Educação: teoria \& prática. Porto Alegre: UFRGS. Programa de Pós Graduação em Informática na Educação, 2002.

PCNs. Parâmetros Curriculares Nacionais: Introdução aos Parâmetros Curriculares Nacionais/ Secretaria de Educação Fundamental. - Brasília: MEC/SEF, 1997.126p. Disponível em http://portal.mec.gov.br/seb/ arquivos/pdf/ciencias.pdf. Acesso em: 15 de out. de 2012.

PEGADA ECOLÓGICA. Que marca quer deixar no planeta? Texto: Mônica Pilz Borba; Coordenação: Larissa Costa e Mariana Valente; Supervisão: Anderson Falcão - Brasília: WWF-Brasil, 2007. Disponível em: http://www.wwf.org.br/ e http://www.footprintnetwork.org/en/index.php/GFN/page/calculators/http:// ecobairro .org.br/site/pegada.pdf, cálculo da pegada ecológica. Acesso em: 03 de nov. de 2012.

PLANETA FAMINTO 2 UM NOVO CAPÍTULO. Documentário brasileiro, 7 min. Disponível em: http:// youtu.be/6zWIAtWXNY0. Acesso em 14 de out. de 2012.

SAMPAIO, M.N; LEITE, L. S. Alfabetização tecnológica do professor. 5. ed. Petrópolis: Vozes, 1999.

SANTOS, Júlio César Furtado. O Papel do Professor na Promoção da Aprendizagem Significativa, 2008. Disponível em: <http://www.pedagogia.com.br/artigos/aprendizagemsig/index.php?pagina=2>. Acesso em 10 out. 2012.

VAN BELLEN, H. M. Indicadores de Sustentabilidade: uma análise comparativa.Tese (Doutorado em Engenharia de Produção) - Universidade Federal de Santa Catarina. CPGEP/UFSC, 250 p., 2006.

\section{ANEXOS}

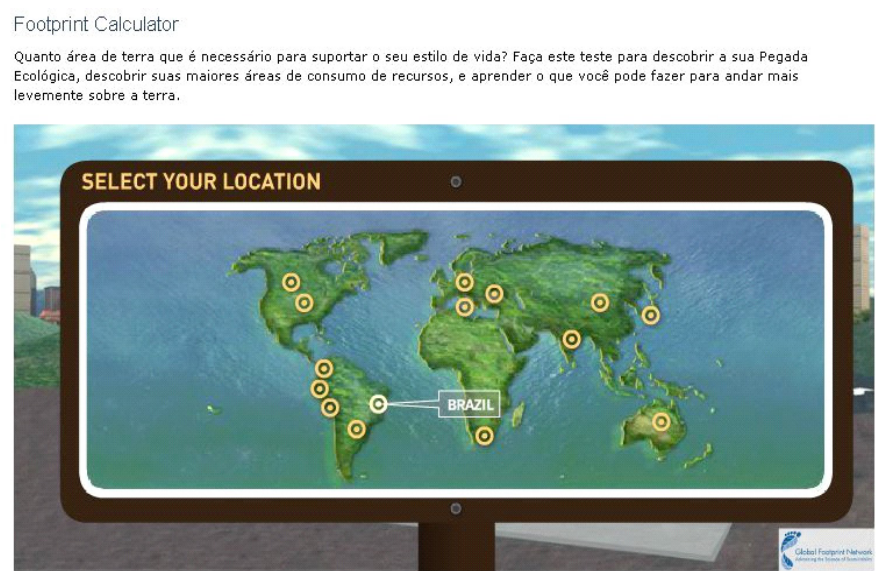

Figura 05: Questionário do cálculo da pegada ecológica. 
Footprint Calculator

Quanto área de terra que é necessário para suportar o seu estilo de vida? Faça este teste para descobrir a sua Pegada Ecológica, descobrir suas maiores áreas de consumo de recursos, e aprender o que você pode fazer para andar mais evemente sobre a terra.

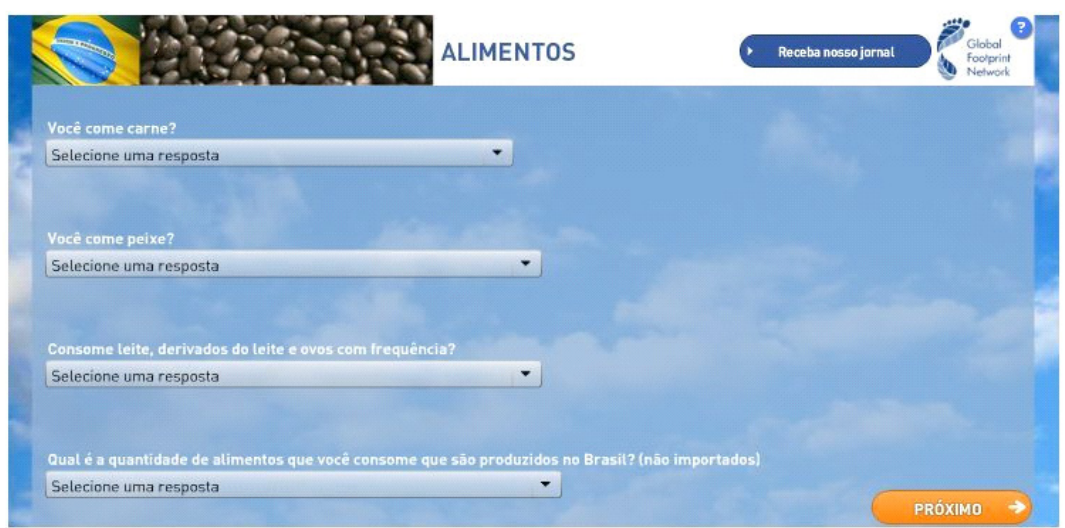

Figura 06: Questionário do cálculo da pegada ecológica.

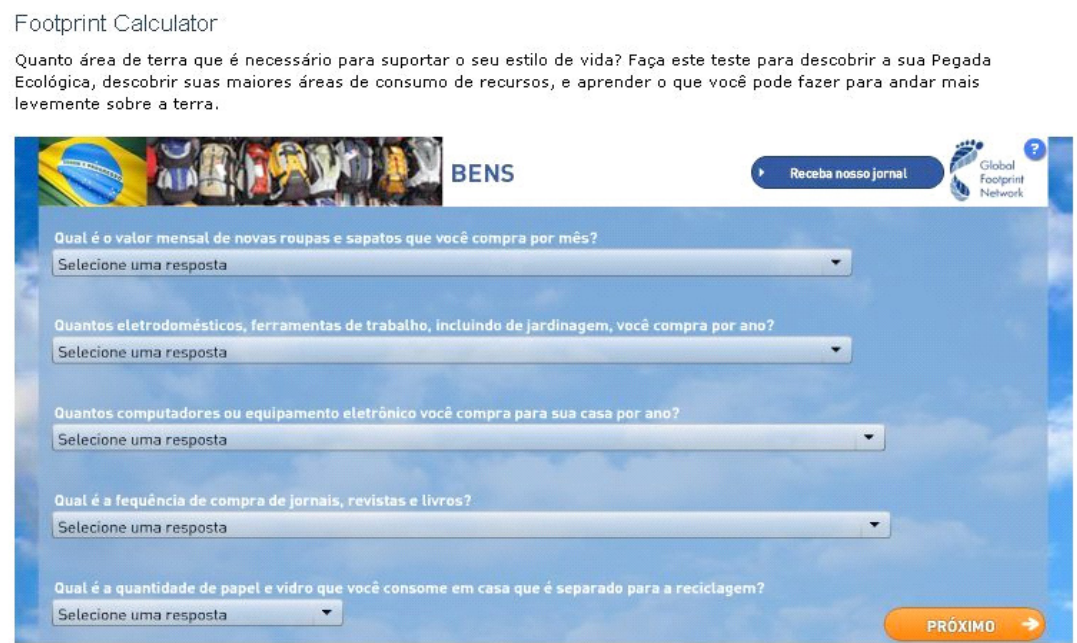

Figura 07: Questionário do cálculo da pegada ecológica.

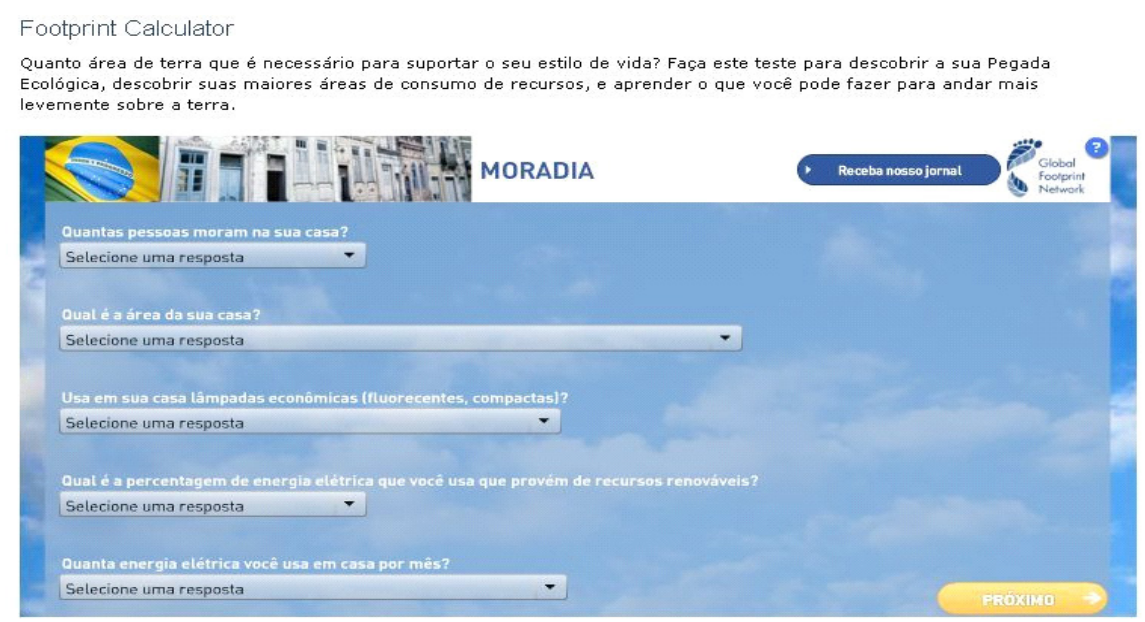

Figura 08: Questionário do cálculo da pegada ecológica. 


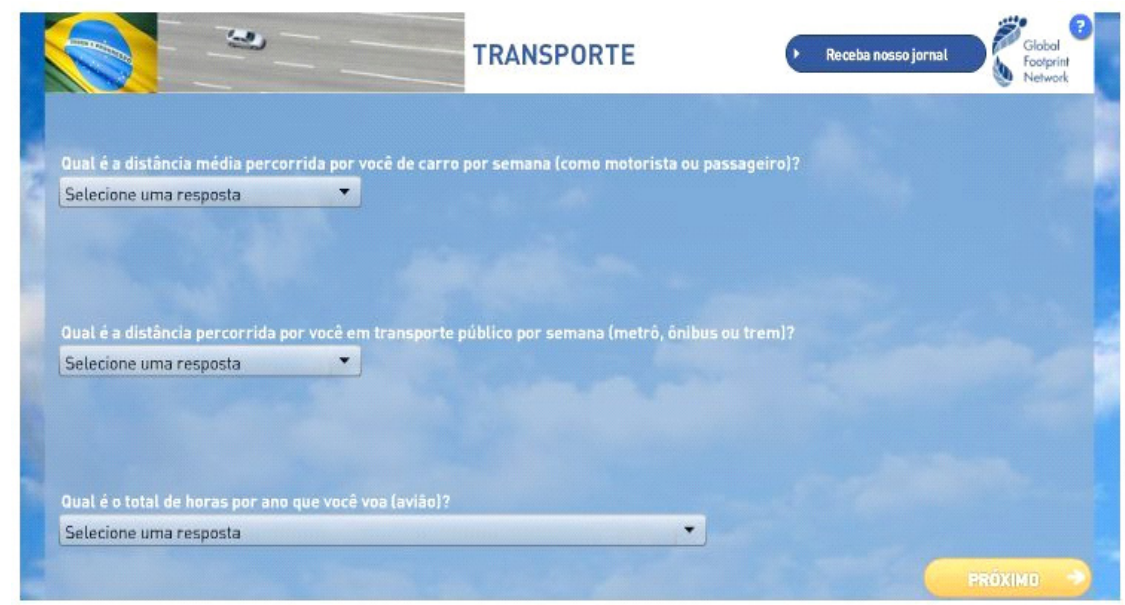

Figura 09: Questionário do cálculo da pegada ecológica.

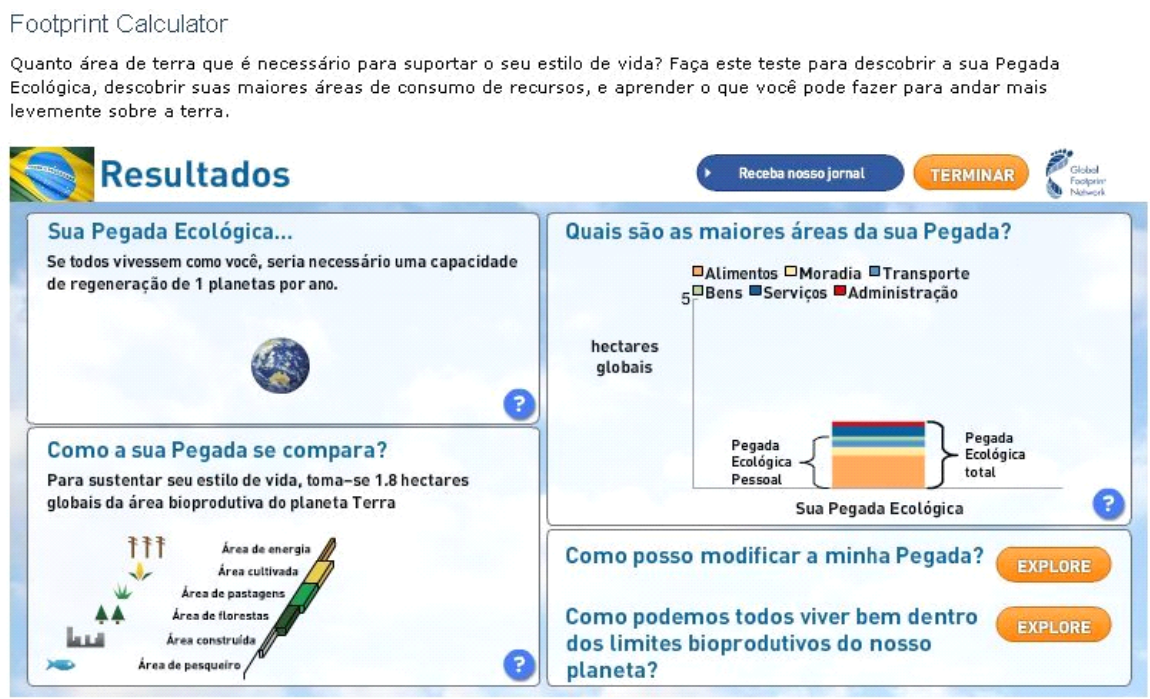

Figura 10: Resultado do cálculo da pegada ecológica.

\section{ANEXO B- VÍDEOS CONSTRUÍDOS PELOS ALUNOS}

\section{VÍDEOS $8^{\circ}$ ANO}

Moda e Consumo: http://www.youtube.com/watch?v=4CpY-TukGEcHYPERLINK "http://www.youtube.com/watch?v=4CpY-TukGEc\&feature=share\%20\&list=UL4CpY-TukGEc"\&HYPERLINK "http://www.youtube.com/watch?v=4CpY-TukGEc\&feature=share $\% 20$ \&list=UL4CpY-TukGEc"feature=share HYPERLINK "http://www.youtube.com/watch?v=4CpY-TukGEc\&feature=share\%20\&list=UL4CpY-TukGEc" \&HYPERLINK "http://www.youtube.com/ watch? $v=4 \mathrm{CpY}$-TukGEc\&feature $=$ share\%20\&list=UL4CpY-TukGEc"list=UL4CpY-TukGEc 


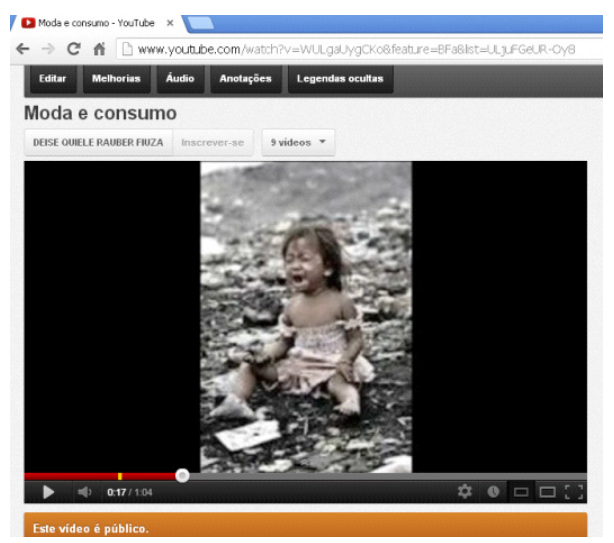

Figura 11: Vídeo construído pelos alunos do $8^{\circ}$ ano.

Consumismo: http://www.youtube.com/watch?v=4CpY-TukGEcHYPERLINK "http:// www.youtube.com/watch? $\mathrm{v}=4 \mathrm{CpY}$-TukGEc\&feature $=\mathrm{BFa} \&$ list $=\%$ 20ULj1cetkX8jwI" \&HYPERLINK "http://www.youtube.com/watch?v=4CpY-TukGEc\&feature $=$ BFa\&list=\%20ULj1 cetkX8jwI"featur $\mathrm{e}=\mathrm{BFaHYPERLINK}$ "http://www.youtube $. \mathrm{com} /$ watch? $\mathrm{v}=4 \mathrm{CpY}-$ TukGEc \&feature $=$ BFa\&list $=\% 20$ ULj1cetkX 8jwI" \&HYPERLINK "http: / / www.youtube.com/watch?v=4CpY-TukGEc\&feature $=$ BFa\&list $=\% 20 U L j 1$ cetkX8jwI”list $=$ ULj1cetkX8jwI

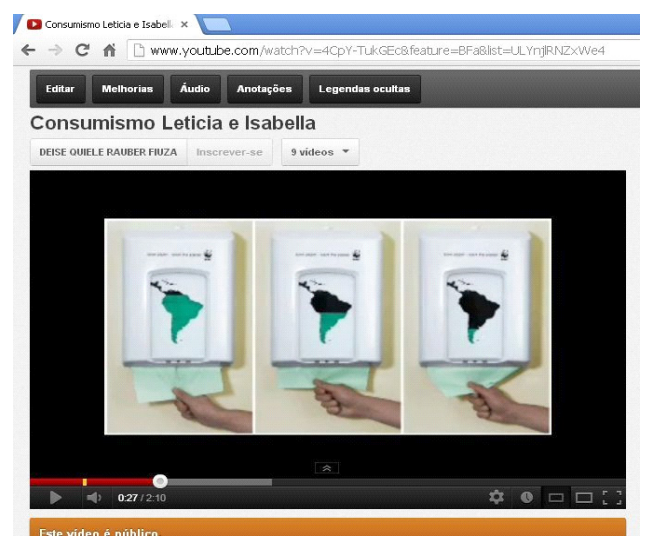

Figura 12: Vídeo construído pelos alunos do $8^{\circ}$ ano.

O Lixo causa destruição: http://www.youtube.com/watch?v=9GOVJbfvFGw HYPERLINK "http://www.youtube.com/watch?v=9GOVJbfvFGw\%20\&feature=BFa\&list=ULUr3Nhup5s8U"\&HY PERLINK "http://www.youtube.com/watch?v=9GOVJbfvFGw\%20\&feature=BFa\&list=ULUr3Nhup 5s8U"feature=BFaHYPERLINK "http://www.youtube $. c 0 m /$ watch?v=9GOVJbfvFGw $\% 20 \&$ feature $=\mathrm{B}$ Fa\&list=ULUr3Nhup5s8U" \&HYPERLINK "http://www.youtube.com/watch?v=9GOVJbfvFGw\%20 $\&$ feature $=\mathrm{BFa} \&$ list $=\mathrm{ULU} 3 \mathrm{Nhup} 5 \mathrm{~s} 8 \mathrm{U}$ "list $=\mathrm{ULUr} 3 \mathrm{Nhup} 5 \mathrm{~s} 8 \mathrm{U}$

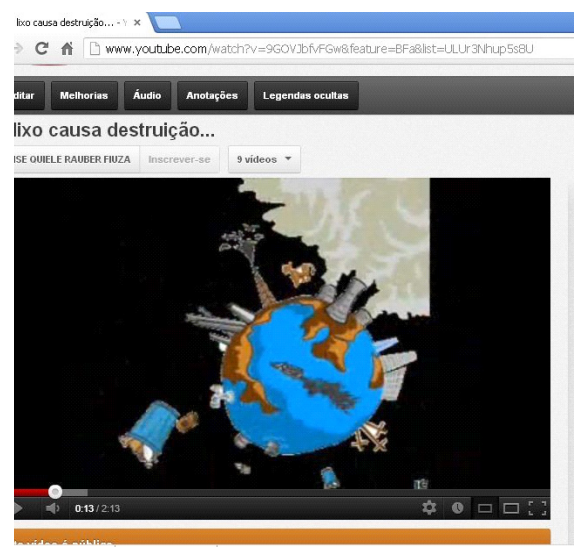

Figura 13: Vídeo construído pelos alunos do $8^{\circ}$ ano. 
Ato de consumir: http://www.youtube.com/watch?v=SMfJoSf3Hh0HYPERLINK "http:// www.youtube.com/watch?v=SMfJoSf3Hh0\&feature=youtu.be" \&HYPERLINK "http://www.youtube. $\mathrm{com} /$ watch?v=SMfJoSf3Hh0\&feature=youtu.be"feature=youtu.be

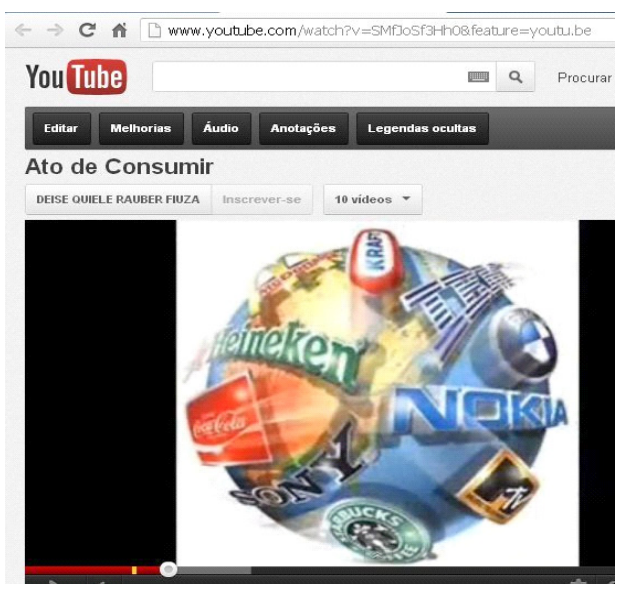

Figura 14: Vídeo construído pelos alunos do $8^{\circ}$ ano.

Consumo induzido: http://www.youtube.com/watch?v=xqOzrZYh_1QHYPERLINK "http:// www.youtube.com/watch?v=xqOzrZYh_1Q\&feature=youtu.be"\&HYPERLINK "http://www.youtube. $\mathrm{com} /$ watch? $\mathrm{v}=\mathrm{xqOz}$ ZYYh_1Q\&feature=youtu.be"feature=youtu.be

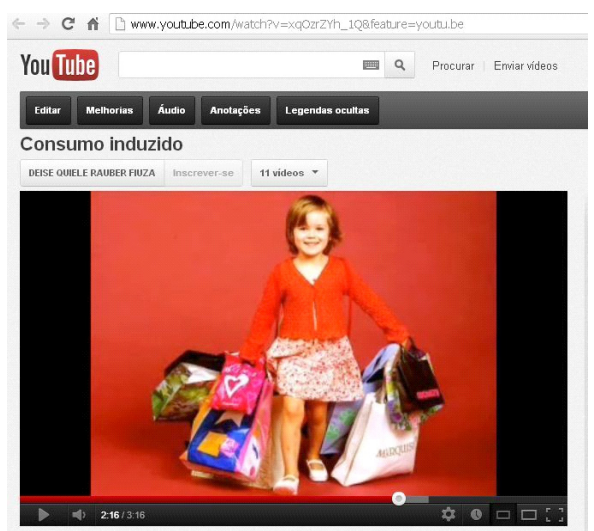

Figura 15: Vídeo construído pelos alunos do $8^{\circ}$ ano.

Vídeos $9^{\circ}$ ano

Lixo e Preservação Ambiental: http://www.youtube.com/watch?v=YnjlRNZxWe4\& eature $=$ autoplay\&list $=$ ULWULgaUygCKo\&playnext $=2$

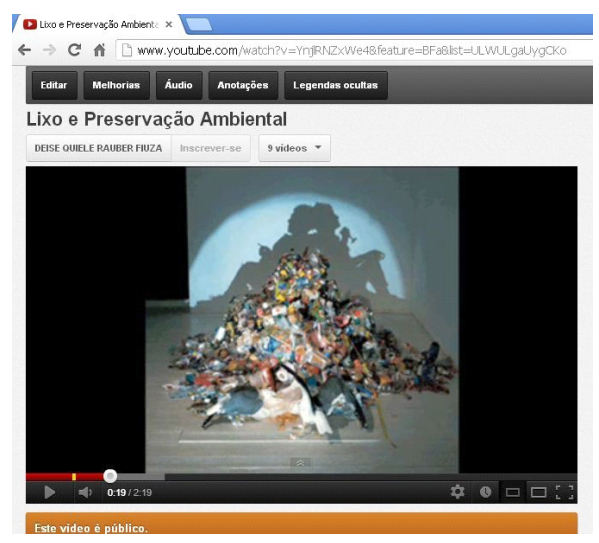

Figura 16: Vídeo construído pelos alunos do $9^{\circ}$ ano. 
Meio Ambiente: http://www.youtube.com/watch?v=j1cetkX8jwIHYPERLINK "http://www. youtube.com/watch? $\mathrm{v}=\mathrm{j} 1$ cetkX8jwI\&feature $=\% 20 \mathrm{BFa} \&$ list $=U L Y$ nj1RNZxWe4" \&HYPERLINK "http://www.youtube.com/watch?v=j1cetkX8jwI\&feature=\%20BFa\&list=ULYnjlRNZxWe4" feature $=$ BFaHYPERLINK "http://www.youtube.com/watch? $\mathrm{v}=\mathrm{j} 1$ cetkX8jwI\&feature $=\% 20 \mathrm{BFa} \&$ list $=\mathrm{ULY}$ n j1RNZxWe4" \&HYPERLINK "http://www.youtube.com/watch?v=j1cetkX8jwI\&feature=\%20BFa\& list=ULYnj1RNZxWe4"list=ULYnjlRNZxWe4

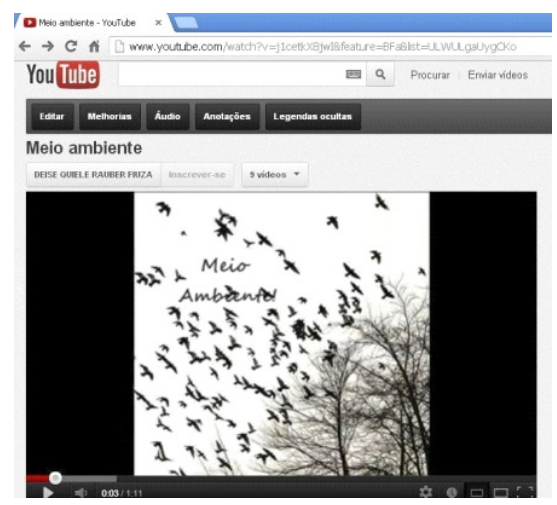

Figura 17: Vídeo construído pelos alunos do $9^{\circ}$ ano.

Degradação do meio ambiente: http://www.youtube.com/watch?v=Ur3Nhup5s8 UHYPERLINK "http://www.youtube.com/watch?v=Ur3Nhup5s8\%20U\&feature=BFa\&list=ULelQCKphEOsE" \&HY PERLINK "http://www.youtube.com/watch?v=Ur3Nhup5s8\%20U\&feature=BFa\&list=ULelQCKph EOsE" feature=BFaHYPERLINK "http://www.youtube.com $/$ watch? $\mathrm{v}=$ Ur3Nhup $5 \mathrm{~s} 8 \% 20$ U \&feature $=$ BFa\&list=ULelQCKphEOsE" \&HYPERLINK "http://www.youtube.com/watch?v=Ur3Nhup5s8\%20 U\&feature=BFa\&list=ULelQCKphEOsE"list=ULelQCKphEOsE

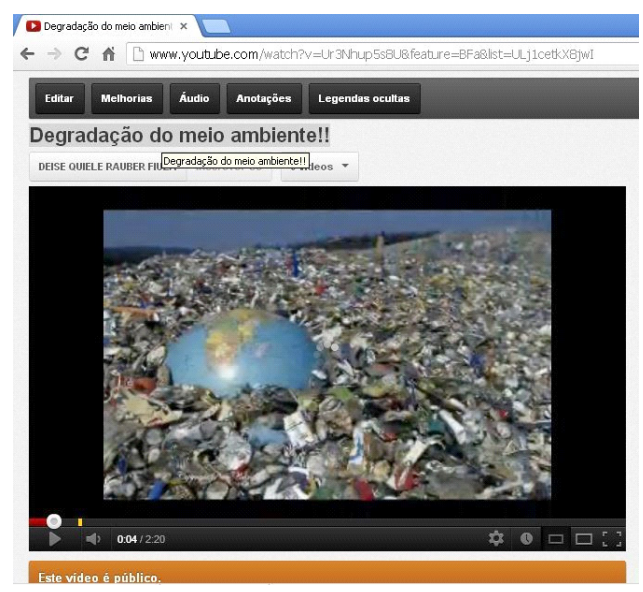

Figura 18: Vídeo construído pelos alunos do $9^{\circ}$ ano.

O que estamos fazendo com o nosso planeta?: http://www.youtube.com/ watch?v=elQCKphEOsEHYPERLINK "http://www.youtube.com/watch?v=elQCKphEOsE\&featu $\mathrm{re}=\mathrm{BFa} \&$ list=UL4CpY-TukGEc" \&HYPERLINK "http://www.youtube.com/watch?v=elQCKphEOs E\&feature $=$ BFa\&list $=U L 4 C p Y-T u k G E c$ " feature $=$ BFaHYPERLINK "http $: / /$ www.youtube. com $/$ wat $\mathrm{ch} ? \mathrm{v}=\mathrm{elQCKphEOsE \& feature=BFa \& list=UL4CpY-TukGEc" \& HYPERLINK} \mathrm{"http://www.youtube.}$ $\mathrm{com} /$ watch?v=elQCKphEOsE\&feature $=$ BFa\&list=UL4CpY-TukGEc"list=UL4CpY-TukGEc 


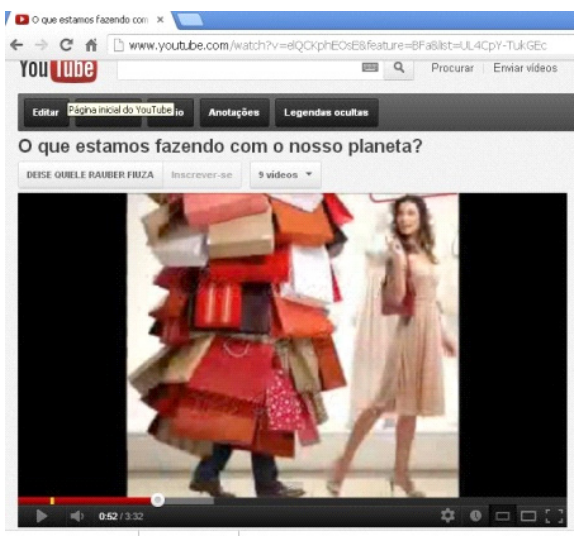

Figura 19: Vídeo construído pelos alunos do $9^{\circ}$ ano. 